\title{
RISCO NUTRICIONAL E COMPLICAÇÕES EM OBESOS HOSPITALIZADOS SUBMETIDOS À CIRURGIA
}

\section{Nutritional risk and complications in hospitalized obese patient submitted to surgery}

\author{
Mariana RASLAN, Lilian Mika HORIE, Maria Cristina Gonzáles BARBOSA-SILVA, \\ Patrícia CAMARGO, Melina CASTRO, Sabrina S. V. GOASTICO, Mariana NASCIMENTO, \\ Maria Carolina Gonçalves DIAS, Ivan CECCONELLO, Dan L. WAITZBERG
}

ABCDDV/572

Raslan M, Horie LM, Barbosa-Silva MCG, Carmargo P, Castro M, Goastico SSV, Nascimento M, Dias MCG, Cecconello I, Waitzberg DL. Risco nutricional e complicações em obesos hospitalizados submetidos à cirurgia. ABCD Arq Bras Cir Dig 2007;20(4):266-9.

RESUMO - Racional - Risco nutricional mede o potencial de morbimortalidade associado ao estado nutricional. A obesidade é um distúrbio nutricional cada vez mais presente em hospitais gerais e pode estar acompanhada de alteração nutricional com conseqüências prejudiciais à evolução clínica do doente, além de ser fator associado a complicações pós-operatórias e morte. Objetivo - Identificar o risco nutricional através da ferramenta de triagem nutricional Malnutrition Universal Screening Tool e verificar o desfecho de complicações pós-operatórias, buscando associação entre risco nutricional e obesidade. Métodos - Avaliaram-se 433 pacientes adultos hospitalizados em enfermarias cirúrgicas variadas, Na admissão hospitalar mediu-se peso e altura corpóreos. Aplicou-se a triagem nutricional após definição da obesidade pelo Índice de Massa Corpórea (Kg/m2) e classificação em grau I (30-34,99 Kg/m2); grau II (35-39,99 Kg/m2); grau III ( $\square 40,00 \mathrm{Kg} / \mathrm{m} 2)$. Os pacientes foram seguidos até a alta hospitalar ou óbito para acompanhar sua evolução clínica. Resultados - Dentre os pacientes avaliados, 17,1\% foram obesos. O risco nutricional nos pacientes obesos foi $23 \%$. Ocorreram complicações em pacientes das enfermarias cirúrgicas de esôfago, vias biliares, vascular, cabeça e pescoço. As complicações apresentadas incluíram infecções pulmonares, de parede abdominal e urinária, fístulas gastrintestinal, biliar e pancreática, insuficiência respiratória, atelectasia lobar, e sangramento pós-operatório. Conclusões - Obesos hospitalizados podem apresentam risco nutricional sendo a obesidade grau I a mais freqüente entre eles em hospital geral. Houve associação positiva entre risco nutricional e freqüência de complicações justificando triagem nutricional na admissão hospitalar como rotina, incluindo pacientes com sobrepeso e obesidade.

DESCRITORES -

\section{INTRODUÇÃO}

De acordo com a Pesquisa de Orçamentos Familiares (POF) do Instituto Brasileiro de Geografia e Estatística (IBGE), com dados de 2002 e 2003, 40\% dos adultos brasileiros apresentam sobrepeso e $11 \%$ obesidade ${ }^{1}$.

A obesidade é um distúrbio nutricional cada vez mais presente no Brasil e no mundo e pode estar acompanhada de risco nutricional com conseqüências prejudiciais à evolução clínica do doente, além de ser fator de causa de complicações médicas seguidas de procedimentos cirúrgicos e mortes precoces ${ }^{2,3,4,5}$. A obesidade leva a distúrbios fisiopatológicos cardiovasculares, respiratórios, endócrinos e metabólicos, os quais influenciam negativamente o resultado peri-operatório ${ }^{3,5}$.

São poucas as publicações associando risco nutricional, desenvolvimento de complicações e obesidade. Sobre a extensão do risco cirúrgico e sua relação com a obesidade os estudos também são escassos ${ }^{4}$.

Embora possam existir cerca de $50 \%$ de desnutridos

Trabalho realizado no Departamento de Gastroenterologia. Disciplina de Cirurgia do Aparelho Digestivo da Faculdade de Medicina da Universidade de São Paulo (FMUSP) - LIM 35, São Paulo, SP, Brasil

Endereço para correspondência: Mariana Raslan, e-mail: marianaraslan@hotmail.com no ambiente hospitalar público, conforme dados do estudo multicêntrico Ibranutri (Inquérito Brasileiro de Avaliação Nutricional Hospitalar), realizado em $2001^{6}$, o sobrepeso e a obesidade ocupam espaço cada vez maior na população brasileira e é de se esperar que em algum momento estes indivíduos necessitem de interisco nutricionalação hospitalar e tratamentos nutricional e medicamentoso adequados.

Risco nutricional mede o maior risco de morbimortalidade associado ao estado nutricional. Tão importante quanto diagnosticar a desnutrição é avaliá-lo em pacientes com situações que podem amplificar os problemas nutricionais ${ }^{7}$.

O risco nutricional é avaliado por questionário que inclui perguntas sobre o estado nutricional atual e gravidade da doença, sendo o primeiro composto das variáveis: índice de massa corpórea (IMC), percentual de perda de peso recente (nos últimos três a seis meses) e ingestão dietética durante a última semana anterior à admissão8 (Figura 1). O IMC, dentro ou acima da normalidade, não detecta o percentual de perda de peso corporal e não prediz isoladamente o risco nutricional. Perda de peso maior que $2 \%$ em uma semana já pode ser considerada significativa, mesmo com IMC normal ou diagnosticando sobrepeso e obesidade ${ }^{9}$. 


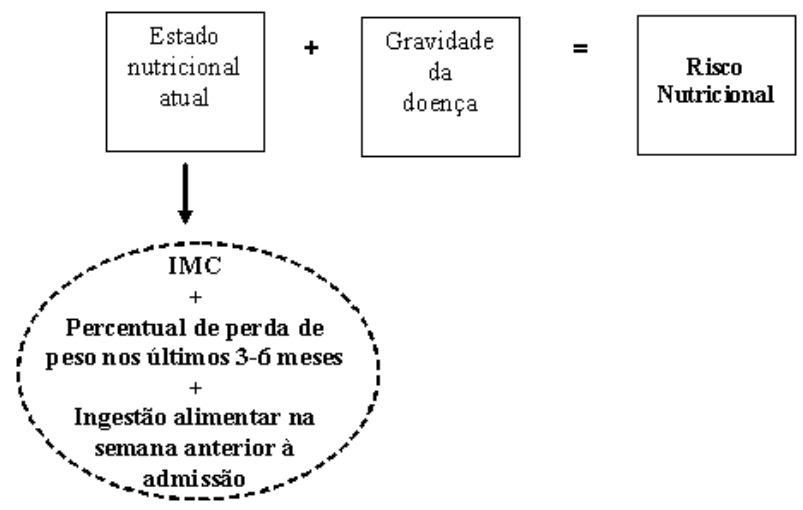

FIGURA 1 - Combinação de elementos para avaliação do risco nutricional

Existem disponíveis várias ferramentas para avaliação de risco nutricional. Entre elas destaca-se a MUST (Malnutrition Universal Screening Tool - Instrumento Universal de Triagem de Desnutrição) desenvolvida pela Associação Britânica de Nutrição Enteral e Parenteral para ser usada por equipe multidisciplinar em adultos de todos os setores de cuidados em saúde. No presente estudo a MUST foi utilizada por ser ferramenta de triagem nutricional capaz de detectar obesidade ${ }^{10}$.

O objetivo do presente trabalho é identificar o risco nutricional através da ferramenta de triagem nutricional MUST e verificar o desfecho de complicações em obesos hospitalizados submetidos a tratamento cirúrgico, buscando estudar eventual associação entre ele e obesidade em cirurgia.

\section{MÉTODOS}

Foram avaliados 650 pacientes com idade acima de 18 anos admitidos em até 48 horas no Instituto Central do Hospital das Clínicas da Faculdade de Medicina da Universidade de São Paulo (ICHC - FMUSP) no período entre fevereiro e agosto de 2007. Destes, 217 foram excluídos por pertencerem a especialidades clínicas e por estarem edemaciados ou com ascite. A amostra analisada foi composta de 433 pacientes adultos hospitalizados em enfermarias de especialidades cirúrgicas variadas, exceto obstetrícia, ginecologia, neurologia, pediatria e pronto socorro.

A lista de interisco nutricionalações hospitalares foi obtida diariamente na Divisão de Arquivo Médico (DAM) do ICHC - FMUSP. Após a separação dos pacientes elegíveis, a identificação dos candidatos ao estudo foi aleatória. Foram incluídos na pesquisa apenas os pacientes que concordaram espontaneamente em participar do presente estudo e assinaram o termo de consentimento livre e esclarecido e que se enquadraram em critérios de inclusão e exclusão pré-estabelecidos.

Mediu-se peso e altura corpóreos em balanças mecânicas e eletrônicas calibradas e com régua medidora acoplada, conforme disponibilidade nas enfermarias do hospital. Aplicou-se a MUST (Quadro 111), após a classificação do $\operatorname{IMC}\left(\mathrm{Kg} / \mathrm{m}^{2}\right)$ em: obesos grau I-OGI- IMC: 30-34,99; obesos grau II -OGIIIMC: 35-39,99; obesos grau III -OGIII- IMC: $\geq 40,008$.

\begin{tabular}{|c|c|c|}
\hline IMC & \multicolumn{2}{|c|}{ Perda de peso nos últimosEfeito da doença } \\
\hline $\begin{array}{l}\text { Pontos } \\
0=>20,0 \\
1=18,5-20,0 \\
2=<18,5\end{array}$ & $\begin{array}{l}3-6 \text { meses } \\
\text { Pontos } \\
0=<5 \% \\
1=5-10 \% \\
2=>10 \%\end{array}$ & $\begin{array}{l}\text { Adicionar } 2 \text { pontos se } \\
\text { houve ou há possibili- } \\
\text { dade de ausência de in- } \\
\text { gestão alimentar por }> \\
5 \text { dias. }\end{array}$ \\
\hline \multicolumn{3}{|c|}{$\begin{array}{l}\text { Somar os pontos. } \\
\text { Risco de desnutrição e guias para manejamento. }\end{array}$} \\
\hline $\begin{array}{l}0=\text { Baixo Risco } \\
\text { Cuidados clínicos de rotina } \\
\text { Repetir a triagem em: } \\
\text { - Hospital: } \\
\text { semanalmente } \\
\text { - Casas de repouso: men } \\
\text { salmente } \\
\text { - Comunidade: anualment } \\
\text { para grupos especiais (ex: } \\
75 \text { anos de idade) }\end{array}$ & $\begin{array}{l}1 \text { = Risco Médio } \\
\text { Observar } \\
\text { - Hospital e casas de re-- } \\
\text { pouso: } \\
\text { Documentar ingestão dieté- } \\
\text {-tica e hídrica por três dias } \\
\text { - Comunidade: } \\
\text { teDocumentar ingestão dieté- } \\
\text { >tica e hídrica pelo menos a } \\
\text { cada 2-3 meses }\end{array}$ & $\begin{array}{l}\geq 2 \text { = Alto Risco } \\
\text { Tratar } \\
\text {-- Informar nutricionista e } \\
\text { equipe multiprofissional } \\
\text {-de terapia nutricional } \\
\text { - Repetir avaliação se- } \\
\text { manalmente } \\
\text { - }\end{array}$ \\
\hline $\begin{array}{l}\text { Todas as categorias de risco: } \\
\text { - Tratar a condição associac } \\
\text { e aconselhamento nas esco } \\
\text { comidas e bebidas quando n } \\
\text { - Registrar categoria de riscc } \\
\text { - Registrar necessidade de } \\
\text { seguir política local }\end{array}$ & $\begin{array}{l}\text { Obesidade: } \\
\text { la e prover ajuda- Registrar a } \\
\text { lhas alimentares,- Para aquele } \\
\text { ecessário das, essas sã } \\
\text { de desnutrição antes do trat } \\
\text { dieta especial e }\end{array}$ & $\begin{array}{l}\text { a presença de obesidade } \\
\text { les com condições associa- } \\
\text { âo geralmente controladas } \\
\text { atamento da obesidade }\end{array}$ \\
\hline
\end{tabular}

QUADRO 1 - Instrumento Universal de Triagem de Desnutrição (Malnutrition Universal Screening Tool - MUST).

Após a realização da $\mathrm{TN}$, os pacientes foram seguidos até o momento da alta hospitalar ou óbito para acompanhar sua evolução clínica, que foi classificada conforme critérios de Buzby et al.12 modificados. A lista de complicações acompanhadas por profissionais médicos está descrita na Quadro 2.

\begin{tabular}{|l|l|l|l|l|}
\hline Leito: & \multicolumn{5}{l|}{ Prontuário no: } \\
\cline { 2 - 5 } \\
Nome: Sexo: Idade:
\end{tabular}

QUADRO 2 - Acompanhamento das complicações infecciosas e não infecciosas de pacientes cirúrgicos. 


\section{RESULTADOS}

Entre os 433 pacientes cirúrgicos avaliados, 17,1\% $(n=74)$ foram obesos. Neles o tempo de interisco nutricionalação hospitalar foi de $9,5 \pm 5,0$ dias, a idade média de $53 \pm 54,5$ anos e o IMC teve média de $34 \pm 32,8$.

$\mathrm{O}$ risco nutricional avaliado na população cirúrgica em seu todo foi $36,25 \%(n=157)$, enquanto nos pacientes obesos foi $23 \%(n=17)$ (Tabela 1$)$.

TABELA 1 - Pacientes obesos quanto ao grau de obesidade, incidência de risco nutricional e complicações infecciosas e não infecciosas.

\begin{tabular}{llll}
\hline & OGI & OGII & OGIII \\
& N (\%) & N (\%) & N (\%) \\
\hline População de obesos & $52(70,3)$ & $16(21,6)$ & $6(8,1)$ \\
Risco nutricional & $11(14,9)$ & $4(5,4)$ & $2(2,7)$ \\
Complicações & $8(10,8)$ & $1(1,3)$ & - \\
Complicações infecciosas & $8(10,8)$ & $1(1,3)$ & - \\
Complicações não infecciosas & $1(1,3)^{*}$ & - & - \\
*Paciente apresentou complicações infecciosas e não infecciosas.
\end{tabular}

Complicações pós-operatórias ocorreram em 7,6 \% $(n=33)$ da população geral e em 9 doentes obesos. As complicações infecciosas predominaram, ocorrendo 93,9 $\%(n=31)$ na população geral e em 6 doentes obesos. Neles, um apresentou complicações infecciosas e não infecciosas ao mesmo tempo e na população geral esse número foi de $2,54 \%(\mathrm{n}=11)$.

No grupo de obesos, houve associação significativa entre o risco nutricional e a ocorrência de complicações $\left(\chi^{2}=7,9\right.$ e $\left.\mathrm{P}<0,005\right)$.

Na Tabela 2 pode-se observar a enfermaria cirúrgica a que os obesos pertenciam, o diagnóstico e a complicação apresentada.

TABELA 2 - Tipo de enfermaria cirúrgica, diagnóstico médico, complicações e número de pacientes obesos.

\begin{tabular}{lllc}
\hline $\begin{array}{l}\text { Enfermaria } \\
\text { cirúrgica }\end{array}$ & \multicolumn{1}{c}{ Diagnóstico médico } & \multicolumn{1}{c}{ Complicações } & N \\
\hline Esôfago & CA de esôfago & $\begin{array}{l}\text { Infecção pulmonar } \\
\text { Fístula gastrintestinal, biliar, }\end{array}$ & $1^{*}$ \\
& & pancreática \\
Vias biliares & Colecistite aguda & Infecção de parede & 2 \\
& & Infecção urinária & 1 \\
Vascular & Estenose de carótida & Insuficiência respiratória & 1 \\
& Aneurisma de aorta & Infecção de parede & 2 \\
& Insuficiência arterial crônica & Atelectasia lobar & 1 \\
Cabeça e pescoço & CA de cabeça e pescoço & Sangramento pós-operatório & 1 \\
\hline
\end{tabular}

* Paciente apresentou mais de uma complicação associada.
Houve um óbito provocado pelo curso natural da doença em paciente portador de câncer de via biliar, que não apresentou risco nutricional na admissão ou complicações pós-operatórias.

\section{DISCUSSÃO}

A doença é o principal fator preditivo de complicações, mas os distúrbios do estado nutricional também são fatores de risco para complicações pós-operatórias. No presente estudo houve associação positiva entre risco nutricional em doentes obesos e freqüência de complicações pósoperatórias.

Pacientes cirúrgicos obesos podem desenvolver complicações pós-operatórias em maior freqüência que não obesos $^{2,13}$. Complicações infecciosas são mais freqüentes em obesos, sendo a infecção de ferida a complicação mais freqüente entre eles submetidos a gastroplastia, úlcera duodenal gástrica, colecistectomia, histerectomia, cesareana, e transplante renal ${ }^{4}$. Quanto maior foi o IMC, maior a freqüência de complicações pós-operatórias nos submetidos à gastrectomia subtotal por câncer ${ }^{14}$.

Observou-se maior freqüência de obesidade nos doentes portadores de afecções cirúrgicas do trato gastrintestinal com concomitante maior risco nutricional. Chama a atenção a elevada freqüência de complicações nos pacientes de obesidade tipo GI em relação aos portadores dos tipos GII e GIII, eventualmente explicada pelo seu menor número..

No presente estudo, complicações infecciosas foram mais freqüentes em doentes obesos do que os não, constituindo causa de maior morbidade. A obesidade tem sido considerada como fator de risco independente para infecção da ferida operatória ${ }^{4,15}$. Os resultados aqui apresentados confirmam a associação positiva significativa entre freqüência de complicações e risco nutricional entre os pacientes obesos cirúrgicos.

Tendo em vista estes resultados sugere-se que triagem nutricional seja implementada de rotina na prática cirúrgica incluindo pacientes com sobrepeso e obesidade, por se tratar de ferramenta de fácil aplicação, sem ônus e com resultados coerentes com a evolução clínica dos doentes.

\section{CONCLUSÕES}

Obesos hospitalizados podem apresentam risco nutricional sendo a obesidade grau I a mais freqüente entre eles em hospital geral. Houve associação positiva entre risco nutricional e freqüência de complicações justificando triagem nutricional na admissão hospitalar como rotina, incluindo pacientes com sobrepeso e obesidade. 
Raslan M, Horie LM, Barbosa-Silva MCG, Carmargo P, Castro M, Goastico SSV, Nascimento M, Dias MCG, Cecconello I, Waitzberg DL. Nutritional risk and complications in hospitalized obese patient submitted to surgery. ABCD Arq Bras Cir Dig 2007;20(4):266-9.

ABSTRACT - Background - Nutritional risk measures the morbid-mortality potential associated to nutritional state. Obesity represents a nutritional disorder frequently in general hospital in nowadays and it may be associated with nutritional alteration, presenting prejudicial consequences to patient clinical outcome, besides being a factor related to postoperatory complications and death. Aim - To identify nutritional risk using Malnutrition Universal Screening Tool and verify the results from postoperatory complications, correlating nutritional risks and obesity. Methods - Total of 433 adults patients hospitalized in general surgical ward were evaluated. At admission, patients were measured on body weight and height. Nutritional screening was applied after obesity definition using Body Mass Index as parameter $\left(\mathrm{Kg} / \mathrm{m}^{2}\right)$, classified in grade I $\left(30-34,99 \mathrm{Kg} / \mathrm{m}^{2}\right)$; grade II $(35$ $\left.39,99 \mathrm{Kg} / \mathrm{m}^{2}\right)$; grade III $\left(>40,00 \mathrm{Kg} / \mathrm{m}^{2}\right)$. Follow-up patients were conducted until patient's discharge or eventual death. Results - Among patients submitted to the study, $17,1 \%$ were considered obese. Nutritional risk in obese patients was verified in $23 \%$. Complications presented during hospital staying were related to esophagus, biliary tract, vascular, head, and neck. Infections complications were pulmonary, abdominal wall, and urinary tract. Complications due to fistulas were located in gastrointestinal tract, biliary tract, and pancreas. Others complications consisted of respiratory insufficiency, lobar atelectasis, and postoperatory bleeding. Conclusion - Hospitalized obese patients might present nutritional risk, being obesity grade I the more frequently one in general hospital. Positive association between nutritional risk and complication frequency was verified in the present study, justifying by the nutritional screening at the hospital admission as routinely, including overweight and obese patients.

HEADINGS - Nutritional status. Body mass index. Obesity.

\section{REFERÊNCIAS}

1. Ministério do Planejamento, Orçamento e Gestão. Instituto Brasileiro de Geografia e Estatística - IBGE. Diretoria de Pesquisas. Coordenação de Índices de Preços Pesquisa de Orçamentos Familiares 2002-2003: Análise da disponibilidade domiciliar de alimentos e do estado nutricional no Brasil. Rio de Janeiro, 2004. Disponível em: http://www.ibge.gov.br/home/estatistica/ populacao/condicaodevida/pof/2002analise/pof2002analise.pdf. Acessado em $10 / 10 / 07$.

2. Choban PS, Heckler R, Burge JC, Flancbaum L. Increased incidence of nosocomial infections in obese surgical patients. Am Surg 1995; 61(11):1001-5.

3. Junior JOCA, Giannini CG, Saragiotto DF. Challenges in perioperative management of morbidly obese patients: how to prevent complications. Rev Bras Anestesiol 2003; 53(2):227-36.

4. Flancbaum L, $\neg$ Choban PS. Surgical Implications of Obesity. Annual Review of Medicine 1998; (49):215-234.

5. Senagore AJ, Delaney CP, Madboulay K, Brady KM, Fazio CVW. Laparoscopic colectomy in obese and nonobese patients. J Gastr Surg 2003; 7(4):558-61.

6. Waitzberg DL, Caiaffa WT, Correia MITD. Hospital malnutrition: the brazilian national survey (Ibranutri): a study of 4000 patients. Nutrition 17:573-580, 2001.

7. Barbosa-Silva MCG, Barros AJD. Avaliação nutricional subjetiva: parte 1Revisão de sua validade após duas décadas de uso. Arq Gastroenterol 2002; 39(3)181-187.
8. Salas-Salvadó J, Rubio MA, Barbany M. Consenso SEEDO (Sociedad Española para el Estudio de la Obesidad) 2007 para la evaluación del sobrepeso y la obesidad y el establecimiento de criterios de intervención terapéutica. Med Clin (Barc) 2007; 128(5):184-196.

9. Cook, Kirk S, Lawrenson S, Sandford S. BAPEN symposium 3 on "from beginners to zimmers". Use of the BMI in the assessment of underisco nutricionalutrition in older subjects: reflecting on practice. Proc Nutr Soc 2005; 64:313-37.

10. Stratton RJ, Hackston A, Longmore D, Dixon R, Price S, Stroud M. Malnutrition in hospital outpatientes and inpatiens: prevalence, concurrent validity and ease of use of the "Malnutrition Universal Screening Tool" (MUST) for adults. Brit J Nutr 2004; 799-808

11. BAPEN (British Association for Enteral and Parenteral Nutrition). The MUST Explanatory Booklet. A guide to Malnutrition universal screening tool (MUST) for adults. Malnutrition Advisory Group (MAG) [cited 2006 Jul 22]. Available from: http://www.bapen.org.uk/pdfs/Must/MUST-Explanatory-Booklet.pdf

12. Buzby GP, Mullen JL, Matthews DC, Hobbs CL, Rosato EF. Prognostic nutritional risk index in gastrointestinal surgery. Am J Surg 1980; 139(1):160-67.

13. Abdel-Moneim RI. The hazards of surgery in the obese. Int Surg 1985; 70(2):101-3.

14. Ilias EJ, Kassab P, Castro OP. Complicações na gastrectomia por câncer e obesidade. Rev Assoc Med Bras 2002; 48(3):1-5.

15. Canturk Z, Canturk NZ, Cetinarslan B, Utkan NZ, Tarkun I: Nosocomial infections and obesity in surgical patients. Obes Res 2003; 11:769-75.

Conflito de interesse: não há

Fonte financiadora: não há

Recebido para publicação em: 04/07/2007 Aceito para publicação em: 29/10/2007 